\title{
Cytomegalovirus Antibodies Amongst Immunocompromised (HIV) Patients At Lagos University Teaching Hospital (LUTH) Idi - Araba, Lagos
}

\author{
A.A.AKI NBAMI ${ }^{1}$, A.SAKANMU ${ }^{2}$, T.A.ADEYEMO ${ }^{2}$, K.O.WRIGHT ${ }^{3}$, M.O.DADA ${ }^{1}$, A.O.DOSUNMU ${ }^{1}$
}

\begin{abstract}
Background: CMV infection constitutes a real risk of pathogenecity in immunocompromised patient. HIV-infected patients who require transfusion are at high risk of developing symptomatic CMV infection when they are transfused with CMV-infected donor blood. This study intends to determine CMV infection risk in correlation with blood transfusion in a population of HIV-infected patients

Methods: The study was carried out amongst clients attending HIV clinic at Lagos University Teaching Hospital (LUTH), who were recruited consecutively. Replacement blood donors of the hospital were recruited as controls. A total of $10 \mathrm{mls}$ of blood sample was collected per consenting participant. The serum obtained from $5 \mathrm{ml}$ of blood was assayed for CMV IgG/lgM using an enzyme- linked immunosorbent assay (ELISA) based Kit whilst full blood count and CD4 cell counts were also performed on the remaining $5 \mathrm{~m} / \mathrm{s}$.

Results: A total of I 29 HIV-infected were studied. All (I00\%) were IgG anti-CMV positive. 8 (6.6\%) were IgM anti$C M V$ positive. This difference was found to be statistically significant $P=0.004,(P<0.05)$. Of I 22 replacement blood donor controls, II 8 (96\%) of them were IgG anti-CMV positive whilst 26 (I9.5\%) of I I were IgM anti-CMV positive.The mean CD4+ cell count of HIV-infected patients was $(234 \pm$ I 73.I8I). Previous history of blood transfusion did not affect CMV antibody positivity directly.

Conclusion: The seroprevalence of CMV is very high in HIV-infected patients, which is comparable to the seroprevalence amongst the general population The use of leukoreduced blood units for anaemic HIV infected patients, is recommended.
\end{abstract}

Keyword: Seroprevalence, CMV IgG/IgM, ELISA, healthy blood donors, HIV-infected patients.

\section{Introduction}

Cytomegalovirus (CMV) is a very frequent infection complicating AIDS. Sexual transmission appears to be the most common route of infection in adults, though CMV can also be spread through oropharyngeal sections, urine, breast milk, and blood1. Most patients with AIDS who develop clinical signs and symptoms of CMV infection probably have reactivation of previous infection rather than primary infection. ${ }^{1}$

CMV is a widely distributed opportunistic agent seen with AIDS. Unlike Pneumocystis jirovecii, which nearly always involves only the lung, CMV can and does involve many organs. The most clinically significant sites of involvement are lung, gastrointestinal tract, brain, and eye. In a large autopsy series, CMV occurred most frequently in adrenal and respiratory tract, followed by the gastrointestinal tract, central nervous system, and eye, infrequently in spleen and genitourinary tract, and rarely in lymph node, skin, liver, bone marrow, or heart.1 The diagnosis of CMV retinopathy, one of the most clinically debilitating complications of CMV infection, is made on fundoscopic examination because of the inability to obtain tissue from the eye. Many patients with CMV retinopathy develop partial or complete blindness. Additional clinical manifestations of CMV infection can include altered mental status, pneumonitis with nonproductive cough, colitis or esophagitis with or without gastrointestinal hemorrhage, adrenal insufficiency, hepatitis, or radiculitis.

The prevalence of HIV/AIDS in Sub Saharan Africa is high but the description of CMV infection as opportunistic infection amongst patients is scanty. Akinsola and Okany et al have reported few cases of CMV retinitis in HIV infected Nigerian. ${ }^{2}$ The HIV patients who developed symptomatic CMV infection may have had the infection for a long time;

1. Department of Haematology and Blood Transfusion, Lagos State University College of Medicine

2. Department of Haematology and Blood Transfusion, Lagos University Teaching Hospital Idiaraba.

3. Department of Community Health and Primary Health Care, Lagos State University College of Medicine.

Correspondence: A.A.Akinbami, E-mail: ajoke_clinic@yahoo.co.uk 
immunosupression by HIV makes the virus to become pathogenic. The higher the prevalence of CMV in the general population, the higher should be the prevalence of CMV infection in the population of HIV infected patients. Anaemia is a common feature of presentation in these patients and is second only to tuberculosis as a cause of admission at the Lagos University Teaching Hospital . ${ }^{3}$ Likelihood of transfusion in HIV infected patient is found to be at least three times higher when compared with transfusion in all other patients in the medical wards . ${ }^{3}$ Thus predisposing them further to the risk of acquiring CMV infection through blood transfusion.

CMV infection constitute a real risk of pathogenecity in immunocompromised patient, it is likely that HIV infected patients who develop CMV infection may have a previous history of blood transfusion. Also HIV infected patients who require transfusion are at high risk of developing symptomatic CMV infection when they are transfused with CMV infected donor blood. This study aims to determine the risk of transfusing patients with blood might result in CMV infection in a population of HIV infected patients (immunocompromised patients).Also to determine the prevalence of CMV infection in HIV infected patient with and without past history of blood transfusion.

The findings from this work may help to develop policy whether CMV screening should be routinely done before transfusing HIV infected patients, or in a case of high seroprevalence of CMV amongst the general population, the use of leukoreduced blood units for anaemic HIV infected patients, may be recommended, since CMV is transmitted through the white blood cell.

\section{Materials and Methods}

This cross sectional study was carried out at the HIV outpatient clinic of the Lagos University Teaching Hospital. An average of 15 new cases were seen each clinic day. All newly registered consenting HIV patients who were treatment naive were recruited into the study from 6th of September to 1st of November 2006 after obtaining the institution's research and ethics committee's approval. Data on sociodemography and history of blood transfusion were obtained by a structured questionnaire. Control subjects were apparently healthy HIV- negative replacement blood donors of the hospital.

Five milliliters of blood was collected into sterile unanticoagulated bottle after obtaining patient's consent. The samples were centrifuged, sera separated into sterile bottles on each collection day for storage at -20oC. Another $5 \mathrm{ml}$ was collected into EDTA bottle for full blood and CD4 counts done on the same day of collection.

The CMV specific IgG/IgM antibodies were studied by commercial kit DIA.PRO Diagnostic Bioprobes CMV IgG/ IgM Elisa Kit (Italy), according to the manufacturer's instructions. All specimens were analyzed using enzyme immunoassay test. The cut-off of the device was set at 0.5 WHO IU/ml (Caliberator 2) by the kit's manufacturer. Samples with a concentration higher than $0.5 \mathrm{WHO} \mathrm{IU} / \mathrm{ml}$ were considered positive for CMV IgG whilst samples with concentration below the cut-off as negative results. IgM test results were interpreted as a ratio of the sample OD $450 \mathrm{~nm}$ and the Cut-off value (S/Co) according to the following table.

\begin{tabular}{ll}
\hline S/Co & Interpretation \\
$<1.0$ & Negative \\
$1.0-1.2$ & Equivocal \\
$>1.2$ & Positive \\
\hline
\end{tabular}

The controls and the calibrators passed the validation check recommended by the manufacturers for both the IgG and the IgM kits.

The descriptive data was given as mean \pm standard deviation (SD). Chi-squared test was used for the analytic assessment. The differences were considered to be statistically significant when the p value obtained was less than 0.05 .

\section{Results}

Response rate was $100 \%$, although, one gender record was not filled by a respondent. Out of the total of $129 \mathrm{HIV}$ - infected patients studied, 87 (67.4\%) were females and 41 (31\%) males. The overall mean age of the HIV patients was 34.81( \pm 7.9 ). The mean age of male HIV-infected patients was 38.25 \pm 7.79 . The mean age of female HIV-infected patients (33.10 \pm 7.6 yrs) was significantly lower than that of the males $\mathrm{p}=0.006$

Out of the 129 subjects studied, 98 were screened for IgG anti-CMV. All the 98 HIV infected patients (8 with and 90 without history of transfusion) were CMV IgG positive (100\%). (Table I) As such, association can not be established between transfusion history and CMV IgG positivity. The mean CD4 counts of HIV patients who were IgG positive was 234.07 \pm 173.78 . (Table II). There were no HIV infected patients who were IgG anti-CMV negative for comparison.

Out of the 129 subjects studied, 120 subjects were screened for IgM anti- CMV. Of these, 112 (93.3\%) were negative and 8 (6.6\%) were positive. (Table I). This prevalence was significantly lower than the $19.5 \%$ found in the controls $\mathrm{\Xi}^{2}=$ 8.26,p $=0.0046$. The mean CD4 counts of HIV patients who were IgM anti-CMV positive was $350 \pm 160.85$ and $239 \pm$ 
192.66 for those who were IgM anti-CMV negative $\mathrm{p}=0.379$ (Table II).

Of the $120 \mathrm{HIV}$ infected patients 8 had been transfused and 7 (87.5\%) of these 8 tested IgM anti CMV negative. (Table III). Of the 112 patient who had not been previously transfused, 105 (93.7\%) tested IgM anti CMV negative. No association was established between history of transfusion and CMV IgM positivity $\mathrm{P}=0.43$. A total of 131 healthy donors were recruited for the study as controls. Of the 131 recruited 122 were screened of IgG anti-CMV and 121 screened for IgM anti-CMV. 118 (96\%) of 122 of the controls were IgG anti-CMV positive whilst 26 (19.5\%) of 121 were IgM antiCMV positive.

Table-I

Anti-CMV IgG/IgM Status amongst Subjects.

\begin{tabular}{lcccc}
\hline Status & IgG & Percent (\%) & IgM & Percent (\%) \\
\hline Positive & 98 & $100 \%$ & 8 & $6.6 \%$ \\
Negative & - & $0 \%$ & 112 & $93.3 \%$ \\
Total & 98 & $100 \%$ & 120 & $100 \%$ \\
\hline
\end{tabular}

Table-II

Anti-CMV IgG/IgM Status and Level of Immunity.

\begin{tabular}{lcccc}
\hline Status & IgG & Mean CD4 Count & IgM & $\begin{array}{c}\text { Mean CD4 } \\
\text { Count }\end{array}$ \\
\hline Positive & 98 & $234.07 \pm 173.78$ & 8 & $350 \pm 160.88$ \\
Negative & 0 & 0 & 112 & $239 \pm 192.66$ \\
\hline Total & 98 & & 120 & \\
\hline
\end{tabular}

Table-III

History of Blood Transfusion and IgG/IgM Status of HIVinfected Patients.

\begin{tabular}{lcccc}
\hline Status & $\begin{array}{c}\text { IgG } \\
\text { Positive }\end{array}$ & $\begin{array}{c}\text { IgG } \\
\text { Negative }\end{array}$ & $\begin{array}{c}\text { IgM } \\
\text { Positive }\end{array}$ & $\begin{array}{c}\text { IgM } \\
\text { Negative }\end{array}$ \\
\hline Positive History & 8 & 0 & 1 & 7 \\
Negative History & 90 & 0 & 7 & 105 \\
\hline Total & 98 & 0 & 8 & 122 \\
\hline
\end{tabular}

\section{Discussion}

A seroprevalence of 100\% IgG anti CMV was documented in this study amongst the subjects and 96\% of the controls. The seroprevalence of CMV IgG of 100\% among healthy blood donors was also found in the study of Krech done at Ibadan Nigeria in 1973. 4 There is therefore, no significant difference in the prevalence of CMV IgG amongst immunocompromised HIV-infected patients as compared with control of immunocompetent blood donors. ${ }^{4}$ A high seroprevalence of between $90-100 \%$ was also found in India amongst immunocompetent subjects in various studies. Atul and Ramanchandrum (2002) found 95\% seroprevalence of CMV IgG amongst blood donors. ${ }^{5}$ Study by Pal, Chitkara, and Krech in 1972 showed 100\% seropositivity for CMV IgG in a population of immunocompetent adults Madhavan and Prakash in 1974 showed that 84-96\% of immunocompetent adults had antibody. 6, 7

A prevalence of $6.6 \%$ was obtained for IgM anti-CMV antibodies amongst HIV-infected subjects in the current study. Neusa and Antonio et al (1998) in Brazil, reported $11.36 \%$ HIV-infected prison inmates were IgM anti-CMV antibodies positive.$^{8}$ In HIV immunocompromised subjects there is the possibility of immune reconstitution syndrome that may result in reactivation of previously silent CMV infection. ${ }^{9}$

CMV-specific antibody of the IgM class has been recognized as a marker of active or recent primary infection with the virus and such donors may have CMV viremia. Recent reports have shown a positive correlation between post transfusion CMV infection and the receipt of blood from IgM anti-CMV antibody positive donors. ${ }^{10,11}$ Although, some authorities are of the opinion that the assertion claiming the individuals with IgM anti-CMV are more likely to transmit the virus than those with IgG anti-CMV is not proven beyond doubts. ${ }^{12}$

Lamberson and McMillan (1988) found that a decreased incidence of Transfusion-associated -CMV (TA-CMV) infection occurred when only blood products negative for CMV IgM were used. ${ }^{13}$ In the present study, there is no significant association between history of blood transfusion and being CMV IgG/ IgM positive amongst HIV-infected patients. Hence previous blood transfusion is not a risk factor for CMV antibody production as seen in this study. This is at variance with the work of Tolpin and Stewart in 1985 that provided the first biochemical and molecular evidence for transfusion associated-CMV infection. ${ }^{14}$ The study reported that monocyte latently infected with CMV represent the primary vector for TA-CMV which can be largely abrogated by transfusing at-risk patients with either seronegative units or blood filtered to remove white blood cells. However, several large studies in greater than 1500 blood donors have failed to confirm these observations Beneke(1985). ${ }^{15}$

CMV infections are important clinical problems in patients with HIV infections and AIDS. The use of CMV- antibody free blood component is indicated for CMV- antibody negative AIDS patients although in this study none of HIVinfected patients was CMV- antibody negative. ${ }^{15}$ 


\section{Conclusion}

The seroprevalence of CMV is very high in HIV-infected patients and also high amongst the general population, the use of leukoreduced blood units for anaemic HIV infected patients, is recommended, since CMV is transmitted through the white blood cell.

\section{Acknowledgements}

This is to thank members of staff of both the blood donor and HIV clinics for the support and cooperation during the recruitment exercise.

\section{Conflict of interest: None}

\section{References}

1. Klatt EC, Shibata D. Cytomegalovirus infection in the acquired immunodeficiency syndrome: clinical and autopsy findings. Arch Pathol Lab Med. 1988; 112:540-544.

2. Akinsola F.B., Okany C.C,. Majekodunmi A.A and. Akinsete I. Ocular Manifestations of HIV Infections in Lagos University Teaching Hospital. The Nigeria Postgraduate Medical Journal 1997.Vol. 4, No, 3, September

3. Akanmu A.S, Esan O.A, Taiwo-Osinubi. P. Transfusion dependent Anaemia. A common indication of admission in HIV-infected patients at the Lagos University Teaching Hospital.4th National Conference on HIV/AIDS. International conference centre Abuja. May 2-5 2004.

4. Krech U. Complement fixing antibodies against CMV in different parts of the world. Bull World Health Organ 1973; 49:103-6.

5. Atul Kothari, V.G Ramachandrum et al. Seroprevalence of Cytomegalovirus among voluntary blood donors in Dehli, India. J Health Popul NUTR 2002 Dec; 20(4):348-351.

6. Pal SR, Chitkara NK, Krech V. Seroepidemiology of cytomegalovirus infection in and around Chandigarh. Indian J Med Res 1972 ; 60:973-8.
7. Madhavan HN, Prakash K, Agarwal SC. Cytomegalovirus infections in Pondicherry: a serological survey. Indian J Med Res 1974; 62: 297-300.

8. Neusa M, Antonio F and Lucilia C. Reseach of antigen and antibodies from Retrovirus, CMV and HBV among prisoners of Pententiary complex of the region of Camprinas,S.P. Brazil.Rev Inst. Med. Trop. S.Paulo.1998,Vol 40.n.4

9. Lentz, E. B., Dock N. L. McMahon, C. A. Fiesthumel S. R. Arnold, C. B and Lamberson. H. V. Detection of anti-body of cytomegalovirus-induced early antigens and comparison with four serologic assays and presence of viruria in blood donors. J. Clin. Microbiol. 1988.26:133-135.

10. Griffiths, P. D. Stagno, S. Pass, R. F. Smith, R. J and. Alford. C. A Infection with cytomegalovirus during pregnancy: specific IgM antibodies as a marker of recent infection. J. Infect. Dis. 1982. 145:647-653.

11. Mc Cullough.J .J. Transfusion transmitted disease. In: Transfusion Medicine. New York. Mc Graw-Hill 1998 Pages 361-386.

12. Lamberson HV, Jr., McMillan JA, Weiner LB, Williams ML, Clark DA, McMahon CA et al . Prevention of transfusion associated cytomegaloviral infection in neonates by screening blood donors for IgM to CMV.J Infect Dis 1988; 157:820-3.

13. Tolpin, M. D., Stewart, J. A Warren, D. Mojica, B. A. Collins M. A, Doveikis, S. A. Cabradilla, C. Schauf, V. Raju, T. and Nelson. K. Transfusion transmission of cytomegalovirus confirmed by restriction endonuclease analysis. J. Pediatr. 1985.107:953-956.

14. Beneke, J. S., Tegtmeier G. E Alter, H. J. Luetkemeyer, R. B. Solomon, R. and. Bayer W. L. Relation of titers of antibodies to CMV in blood donors to the transmission of cytomegalovirus infection. J. Infect. Dis. 1984. 150:883888.

15. Mc Cullough.J .J. Transfusion transmitted disease. In: Transfusion Medicine. New York. Mc Graw-Hill 1998 Pages 361-386. 